\title{
NIRCam: Development and Testing of the JWST Near-Infrared Camera
}

\author{
Thomas Greene $^{a}$, Charles Beichman ${ }^{b}$, Michael Gully-Santiago $^{c}$, Daniel Jaffe ${ }^{c}$, Douglas Kelly ${ }^{d}$, \\ John Krist ${ }^{e}$, Marcia Rieke ${ }^{d}$, and Eric H. Smith ${ }^{f}$ \\ ${ }^{a}$ NASA's Ames Research Center, MS 245-6, Moffett Field, CA, USA; \\ ${ }^{b}$ NExScI, Caltech 100-22, Pasadena, CA, USA; \\ ${ }^{c}$ University of Texas Department of Astronomy, Austin, TX, USA; \\ ${ }^{d}$ Steward Observatory, University of Arizona, Tucson, AZ, USA; \\ ${ }^{e}$ Jet Propulsion Laboratory, 4800 Oak Grove Drive, Pasadena, CA, USA; \\ fLockheed Martin Advanced Technology Ctr., 3251 Hanover St., Palo Alto, CA USA
}

\begin{abstract}
The Near Infrared Camera (NIRCam) is one of the four science instruments of the James Webb Space Telescope (JWST). Its high sensitivity, high spatial resolution images over the $0.6-5 \mu \mathrm{m}$ wavelength region will be essential for making significant findings in many science areas as well as for aligning the JWST primary mirror segments and telescope. The NIRCam engineering test unit was recently assembled and has undergone successful cryogenic testing. The NIRCam collimator and camera optics and their mountings are also progressing, with a brass-board system demonstrating relatively low wavefront error across a wide field of view. The flight model's long-wavelength Si grisms have been fabricated, and its coronagraph masks are now being made. Both the short $(0.6-2.3 \mu \mathrm{m})$ and long $(2.4-5.0 \mu \mathrm{m})$ wavelength flight detectors show good performance and are undergoing final assembly and testing. The flight model subsystems should all be completed later this year through early 2011, and NIRCam will be cryogenically tested in the first half of 2011 before delivery to the JWST integrated science instrument module (ISIM).
\end{abstract}

Keywords: JWST, NIRCam, infrared, astronomical instruments, space telescopes

\section{INTRODUCTION}

NIRCam, the Near-Infrared Camera, is perhaps the most critical of four science instruments to be launched with the James Webb Space Telescope (JWST). Like the other instruments (FGS / tunable filter, NIRSpec, MIRI), NIRCam will be used to collect large amounts of data essential for achieving JWST's science goals. ${ }^{1}$ However, NIRCam is unique in that it will also be used to align the JWST optical telescope element (OTE) and phase its primary mirror segments, using dispersive elements in its pupil wheels. ${ }^{2,3}$ NIRCam also has unique design and materials features, including two completely redundant optics modules, a Be optical bench, and $102048 \times 2048$ pixel HgCdTe HAWAII-2RG detector arrays. It also uses refractive collimator / camera optics with dichroic beasmsplitters to allow simultaneous imaging of $0.6-2.3 \mu \mathrm{m}$ and $2.4-5.0 \mu \mathrm{m}$ wavelengths over a $2.2 \times 4.4$ arc-minute field of view (using both the $\mathrm{A}$ and $\mathrm{B}$ optics modules). In addition to providing wide-field imaging capabilities with either 32 milli-arcsecond $(\lambda=0.6-2.3 \mu \mathrm{m})$ or 64 milli-arcsecond $(\lambda=2.4-5.0 \mu \mathrm{m})$ projected pixels, NIRCam can also provide coronagraphic high contrast imaging and slitless grism spectroscopy modes. ${ }^{4,5}$ A solid rendering of one of the optics modules, including major components and the optical path, is shown in Figure 1.

The scientific program and design features of NIRCam have been well described elsewhere ${ }^{6,7}$ and will not be elaborated upon here. Instead, we shall provide details of the recent NIRCam engineering test unit (ETU) test campaign, describe the development status of key flight unit subsystems, and explain upcoming plans for the flight model integration and testing.

Further author information: (Send correspondence to T.G.)

T.G.: E-mail: tom.greene@nasa.gov, Telephone: +1 6506045520

Space Telescopes and Instrumentation 2010: Optical, Infrared, and Millimeter Wave, edited by Jacobus M. Oschmann Jr., Mark C. Clampin, Howard A. MacEwen, Proc. of SPIE Vol. $7731,77310 \mathrm{C} \cdot$ (C) 2010 SPIE · CCC code: 0277-786X/10/\$18 - doi: 10.1117/12.856615

Proc. of SPIE Vol. 7731 77310C-1 


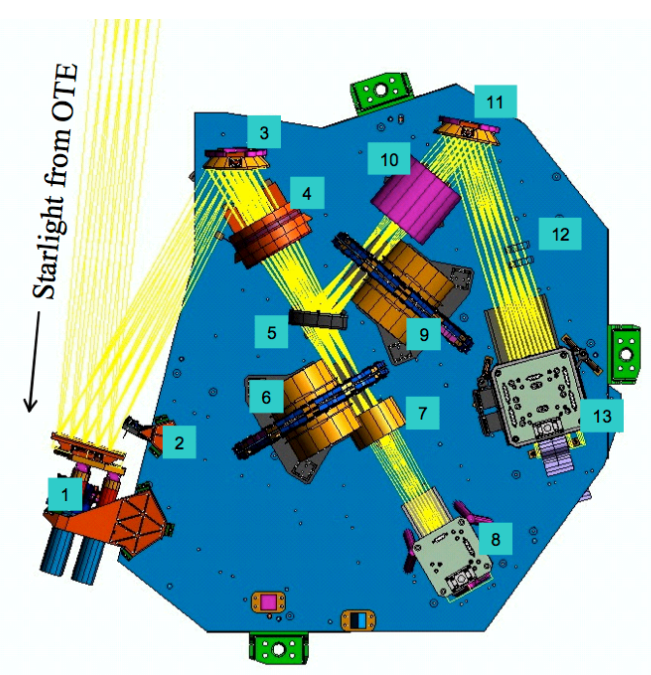

\begin{tabular}{|l|l|}
\hline 1 & Pick-Off Mirror Assembly \\
\hline 2 & Coronagraph \\
\hline 3 & First Fold Mirror \\
\hline 4 & Collimator Lens Group \\
\hline 5 & Dichroic Beamsplitter \\
\hline 6 & Long Wave Filter Wheel Assembly \\
\hline 7 & Long Wave Camera Lens Group \\
\hline 8 & Long Wave Focal Plane Housing \\
\hline 9 & Short Wave Filter Wheel Assembly \\
\hline 10 & Short Wave Camera Lens Group \\
\hline 11 & Short Wave Fold Mirror \\
\hline 12 & Pupil Imaging Lens \\
\hline 13 & Short Wave Focal Plane Housing \\
\hline 14 & ICE Interface Panel \\
\hline 15 & FPE Interface Panel \\
\hline
\end{tabular}

Figure 1. Solid model rendering of the NIRCam optical bench, showing optical components and rays. Major subsystems are identified with numbers and named in the table on the right.

\section{ENGINEERING TEST UNIT}

The NIRCam ETU is composed of a single NIRCam optics module that is populated with only short wavelength optics, several filters, no coronagraph components, and a single $2.5 \mu \mathrm{m}$ cutoff sensor chip assembly (SCA) detector; it is shown in Figure 2. The ETU was installed in a cryogenic vacuum chamber (Red Chamber) at the Lockheed Martin Advanced Technology Center where it underwent testing in early 2010. The optical metrology assembly (OMA) was located exterior to the test chamber and was used as ground support equipment for the testing. Initial tests intended to check the internal alignment of the ETU and the OMA were scrubbed because the optical fibers used to send light from the NIRCam camera focal plane (using a surrogate focal plane array) darkened excessively at cryogenic temperatures. Instead, the OMA was used to simulate the JWST OTE and to inject near-IR light into the chamber and the ETU. The ETU detected this radiation with its single Teledyne HAWAII2-RG near-IR detector with a matching application specific integrated circuit (ASIC) support chip to control the device as well as amplify and digitize its output signals.

The cryogenic ETU tests were completed in April 2010 and were generally very successful. Flat field exposures illuminated by a lamp in the test chamber showed very little unexpected vignetting or illumination gradients. However, the flat field source internal to the $\mathrm{ETU}^{8}$ produced images with a large amount of structure, and the internal flat field illumination system is being redesigned for the NIRCam flight model. The detector linearity and total noise (read-noise, dark current, and background noises) were measured to be consistent with previous tests of the ETU detector with discrete control, preamplifier, and analog-to-digital electronics. This result demonstrates that the ETU detector plus ASIC performance is dominated by the previously measured detector performance and not limited by the ASIC.

The optical performance of the ETU plus OMA system was generally excellent. Figure 3 shows an in-focus point-source image (labeled 0). Several out of focus images, created by selecting NIRCam's various weak lenses, are also displayed with the focus displacement in waves given in each sub-panel. The measured pupil shape, diffraction pattern, and image ghosts were consistent with expected ETU performance.

ETU testing of the Dispersed Hartmann Sensor elements in the NIRCam pupil wheel produced spectral segments with the wavelength coverage and dispersion sufficient to measure the JWST segment and OTE optical alignment errors. The pupil imaging lens (PIL) assembly was used to produce images of the simulated OTE pupil on the NIRCam detector. During cryogenic testing, the primary coil for engaging the PIL developed an open circuit and could not be energized; deployment of the PIL was successfully carried out using the secondary coil instead. Optical distortion in the pupil images revealed that a cylindrical lens in the PIL assembly was not 


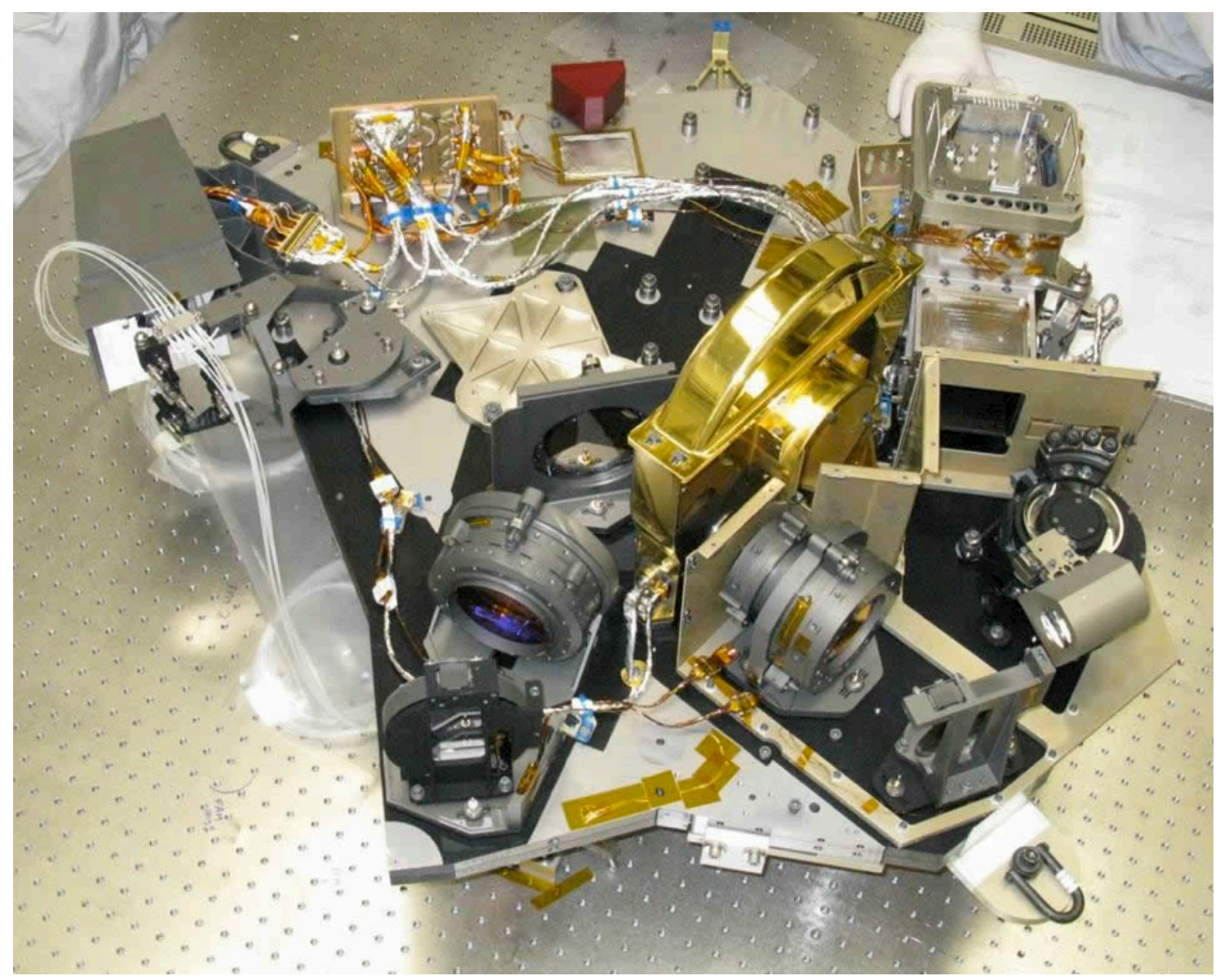

Figure 2. NIRCam ETU optical bench assembly at the Lockheed Martin Advanced Technology Center in February 2010, prior to cryogenic testing. The major subassemblies of this intentionally underpopulated bench can be identified by comparison to the solid model shown in Figure 1.

fabricated correctly, and this will be remedied in the flight model. These cryogenic ETU tests were run over 8 days, during which there were no anomalies or crashes with the prototype NIRCam flight software. The ETU has been shipped to NASA's Goddard Space Flight Center where it will be used to verify instrument interfaces and integration and test processes with the JWST integrated science instrument module (ISIM).

\section{FLIGHT MODEL DEVELOPMENT STATUS}

NIRCam flight model (FM) subsystems and components are now being fabricated, and the FM is scheduled to be integrated later this year. We now report on the development status of some of the more challenging and unique NIRCam FM subsystems.

\subsection{Collimator and Camera Optics}

NIRCam is required to have a low amount of internal optical wavefront error (WFE) because it will be used as the Observatory wavefront sensor for aligning primary mirror segments and the OTE in general. Tests of a pathfinder collimator and camera optical system in 2008 showed WFE in excess of twice the original $69 \mathrm{~nm}$ WFE requirement at some field positions. Optical performance varied little between warm and cold tests, and subsequent analysis has shown that this high WFE was largely due to poor alignment of the individual lenses.

The lens manufacturing tolerances were tightened, the lens mount design was modified, and a new lens alignment technique was developed. The new alignment technique evaluates the WFE at multiple field positions of the triplet system before the individual lens cells are bolted into place. A brass-board triplet camera system incorporating these improvements has been assembled and has passed vibration testing. It was cold cycled to 35 $\mathrm{K}$ twice in early 2010, and its measured system wavefront error was found to match predicted performance very 


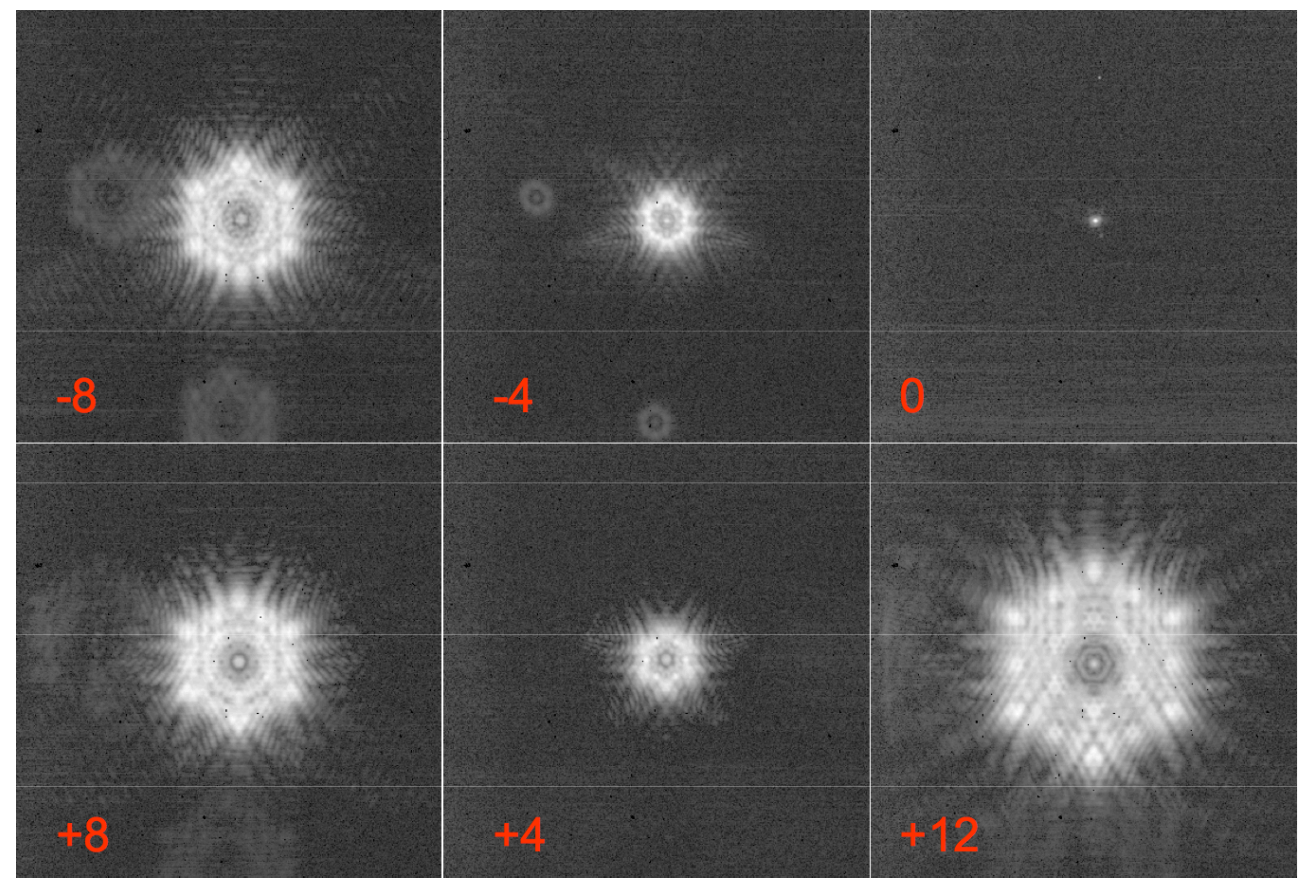

Figure 3. NIRCam OMA + ETU in-focus and out of focus images. The number in each panel indicates the number of waves displaced from focus using weak lenses in the ETU pupil wheel, with 0 corresponding to the in-focus image.

closely at both warm and cold temperatures. Twenty-five field points distributed over an area slightly larger than the effective NIRCam field were measured at 0.785 and $1.55 \mu \mathrm{m}$ wavelengths. Based on this testing and a Monte Carlo analysis, it is expected that over $80 \%$ of the NIRcam field of view will have total wavefront error less than $69 \mathrm{~nm}$. All flight model lenses have also been fabricated and are being coated. Thus the FM optical system development is now advancing well, and we expect that the FM will have very good optical performance.

\subsection{Coronagraph masks}

The NIRCam coronagraphs consist of several spot and wedged-shaped half tone gray-scale coronagraphic focal plane masks accompanied by Lyot masks and wedges in the NIRCam pupil wheels. ${ }^{4}$ The spot and wedge-shaped occulters are designed to provide inner working angles (IWAs) of $2-6 \lambda / \mathrm{D}$ angular radii (where $\mathrm{D}=6.5-\mathrm{m}$ JWST aperture) over a wavelength range of $\lambda=2.1-4.6 \mu \mathrm{m}$. Although the JWST OTE is not optimized for highly stable or high contrast images, these coronagraphs should provide moderately high contrast performance as shown in Figure $4 .{ }^{9}$ When considered with their relatively long $3-5 \mu \mathrm{m}$ near-IR operating wavelengths, these coronagraphs will be able to detect cool gas giant exoplanets planets that are undetectable with higher contrast, smaller IWA coronagraphs on larger ground-based telescopes. ${ }^{9}$ The NIRCam coronagraphic mask patterns are being etched from substrates at the JPL microdevices laboratory, and a close up of one trial spot mask is shown in Figure 5. More details on the status of mask development is reported in another paper in this volume. ${ }^{10}$

\subsection{Long-wave grisms}

The University of Texas has recently delivered a set of six diffraction-limited grisms for NIRCam. ${ }^{11}$ The grism grooves are micromachined in silicon, a high refractive index $(\mathrm{n}=3.4)$ material, with good transmission from $\lambda=3-5 \mu \mathrm{m}$. The blazed grisms have $48 \mathrm{~mm}$ diameters, pitch 0.65 grooves $\mathrm{mm}^{-1}, 5.8^{\circ}$ blaze angles, and $6.1^{\circ}$ prism wedge angles. These properties deliver the $3.7 \mu \mathrm{m}$ first order blaze wavelength undeviated through the grism. The grisms will provide resolving power $R \sim 1800$ and have $42 \mathrm{~mm}$ clear aperture diameters. At the $3.7 \mu \mathrm{m}$ blaze wavelength, the Si surfaces are flat to about $\lambda / 100$ peak-to-valley over the grating's center $31 \mathrm{~mm}$ diameter (the NIRCam pupil size). All measured optical properties of the gratings meet or exceed their 


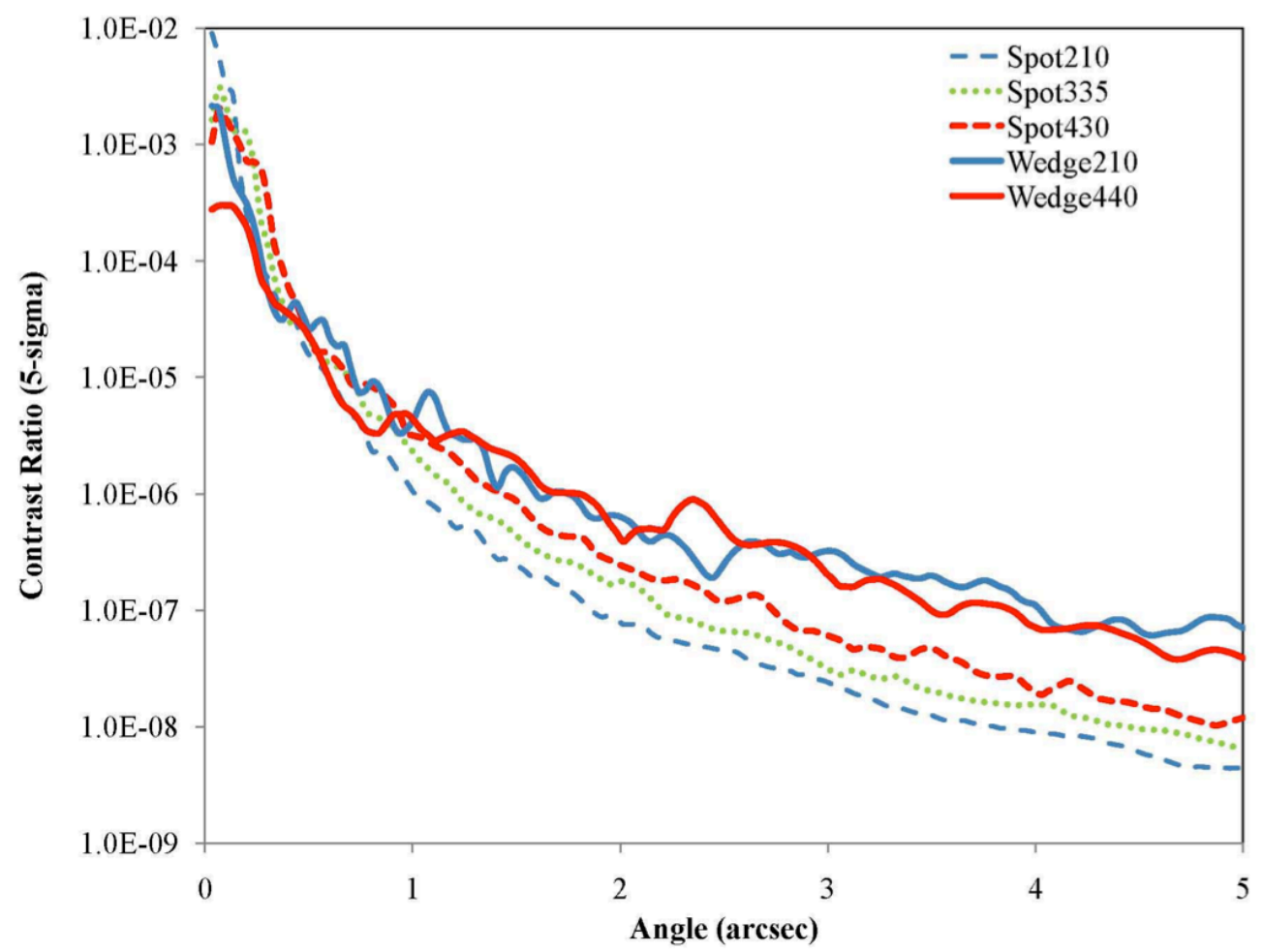

Figure 4. The contrast ratio $(5 \sigma)$ as a function of off-axis angle is shown for the various NIRCam coronagraph masks assuming subtraction of two rolls $\left(+5^{\circ}\right.$ and $\left.-5^{\circ}\right)$ for speckle suppression. A position offset error of 10 mas and a wavefront error of $10 \mathrm{~nm}$ between rolls has been assumed. Estimated performance of the two wedges are shown as solid lines $(4.60 \mu \mathrm{m}$ in red, $2.10 \mu \mathrm{m}$ in blue), and the performance of the three spots are shown as dash/dotted lines $(4.30 \mu \mathrm{m}$ in red, $3.35 \mu \mathrm{m}$ in green and $2.10 \mu \mathrm{m}$ in blue). ${ }^{9}$

requirements. To reduce the large ( $30 \%$ ) Fresnel losses at the vacuum-Si interface, all of the grisms were antireflection (AR) coated on their flat grism entrance surfaces. AR coatings have also been applied to the grooved surfaces of test parts, and these coatings were found to have no adhesion flaws after ten rapid cryogenic cycles. The grooved surfaces of two flight grisms will likely be AR coated given this excellent coating adhesion. When both grism sides are coated, we expect to yield blaze efficiencies close to $75 \%$, as measured in a similar trial part. Four grisms will be flown in NIRCam, and one of the delivered parts is shown in Figure 6.

\subsection{Detectors}

Each of the two NIRCam short-wave (SW) focal plane assemblies (FPAs) contain $42048 \times 2048$ pixel substrate removed HgCdTe Teledyne HAWAII-2RG SCA detectors with $2.5 \mu \mathrm{m}$ long wavelength cutoffs. A qualification SW FPA was assembled in 2008 and underwent successful performance and qualification testing. The detectors were flat to within $\pm 40 \mu \mathrm{m}$, and the SCAs were located within $100 \mu \mathrm{m}$ of their nominal positions at $\mathrm{T}=35 \mathrm{~K}$. There was less than $50 \mu \mathrm{m}$ movement between pre- and post-vibration testing. The two flight SW FPAs have been assembled and have undergone performance testing. They are currently undergoing environmental tests. One SW flight FPA, SFMB, is shown in a test dewar in Figure 7.

The total read and dark current noise from the combined SW SCA plus ASIC system was measured to be approximately 7.5 electrons over $850 \mathrm{~s}$ integrations. These integrations consisted of 80 detector reads with signals measured from the slopes of integration ramps. These measurements were made at ASIC and detector temperatures of $38.5-40 \mathrm{~K}$. The long-wave (LW; $\lambda=5 \mu \mathrm{m}$ cutoff) FPAs are still being assembled at the University of Arizona. The flight LW SCAs have been selected, but their performance has not yet been measured using ASICs. 


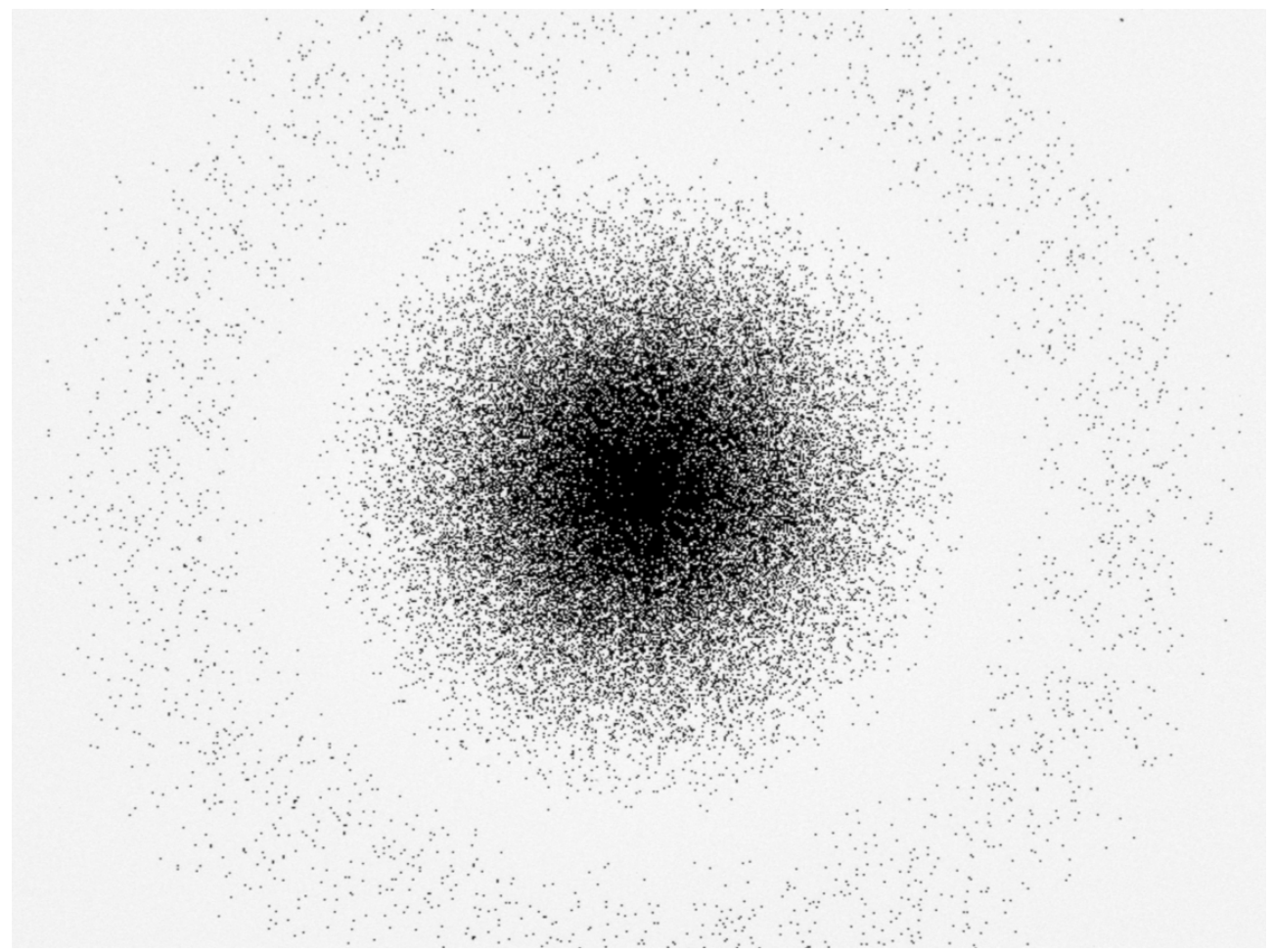

Figure 5. Magnified micrograph image of a NIRCam medium occulting spot.

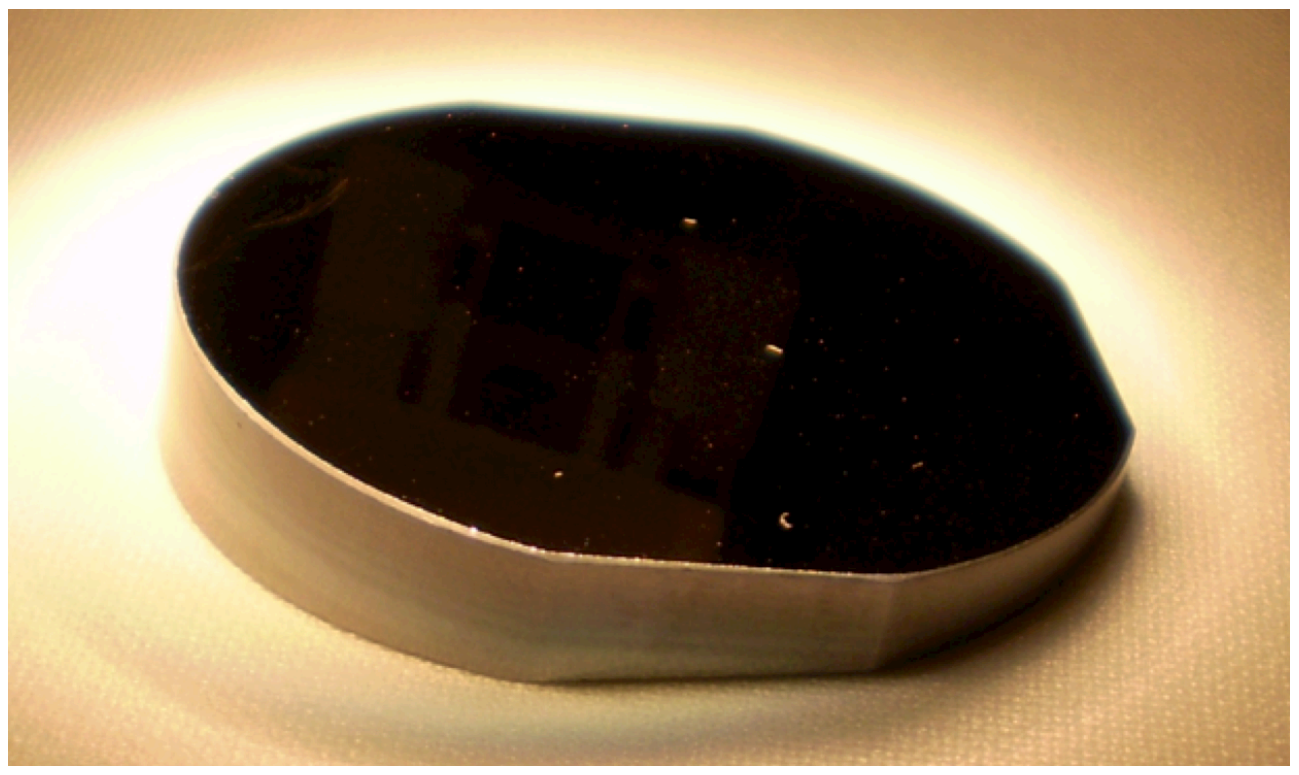

Figure 6. Photograph of a NIRCam Si grism, part number A6F. 


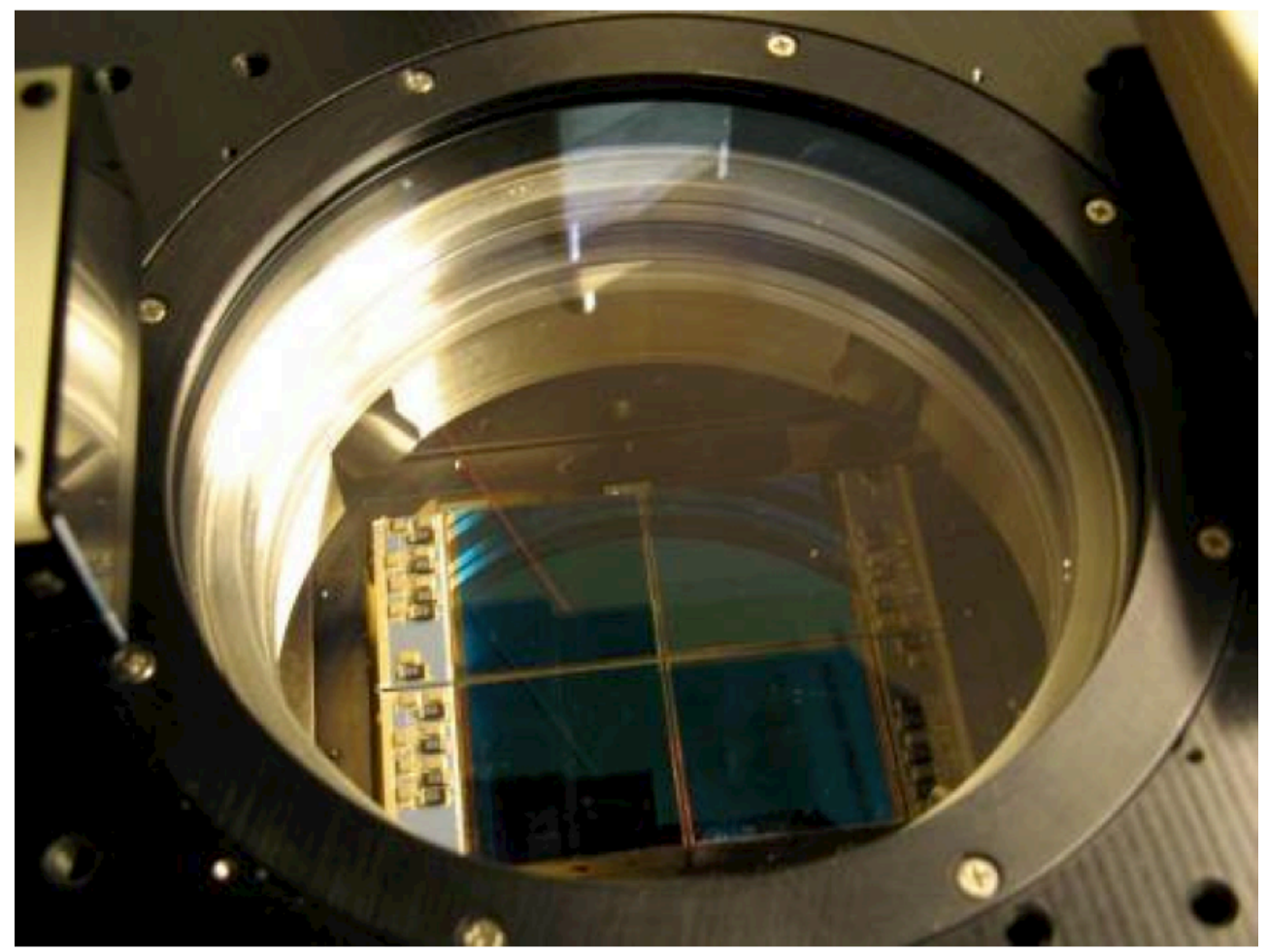

Figure 7. Photograph of the NIRCam flight SW FPA part SFMB installed in a test dewar. The patterns seen on the 4 SCAs are due to reflections on the detectors and are not imperfections of their surfaces.

The SW and LW detectors were found to have latent images that decay with two distinct time constants after saturated exposures. The initial latent was found to have a strength of under $10^{-3}$ of the initial saturated exposure and then decayed exponentially with a time constant of approximately $1000 \mathrm{~s}$. Another, longer latent decay time constant $(\sim 10,000 \mathrm{~s})$ was also observed for heavily saturated exposures, approximately 4 times full well or stronger. However, the latents did not increase in strength for fluxes greater than $2-3$ times saturation. The strength of the latents was also found to be related to the integration time of the saturated exposure and not simply the number of photons incident during an exposure; the initial latent image amplitudes of longer exposures were found to be up to twice as strong as shorter exposures made with the same final saturation level. This latent image behavior is similar to that found for $\lambda=1.7 \mu \mathrm{m}$ cutoff HAWAII-2RG devices tested for the SNAP mission concept. ${ }^{12}$

\section{UPCOMING FLIGHT MODEL INTEGRATION AND TEST ACTIVITIES}

We now discuss highlights of the remaining milestones and activities that must be completed before delivery of the NIRCam FM. Integration and testing of the SW and LW detectors will continue through this summer, with the final FPAs expected to be completed in August. The flight instrument control electronics and the focus and alignment mechanism (FAM) are also expected to be delivered then, followed by completion of the flight optics deliveries. The focal plane electronics will then be delivered, followed by the completion of the filter wheel assembly. Cryogenic testing of the FM is expected to begin before the end of 2010 using an interim release of the flight software; the final flight software is expected to be delivered in the spring of 2011. The first FM comprehensive performance test will be completed in spring 2011 or later, and the NIRCam pre-ship review is currently scheduled for the summer of 2011.

A schematic of the FM test sequence is shown in Figure 8. This shows the significant number of performance and environmental tests that must be successfully completed before delivery of NIRCam. Given the significant 


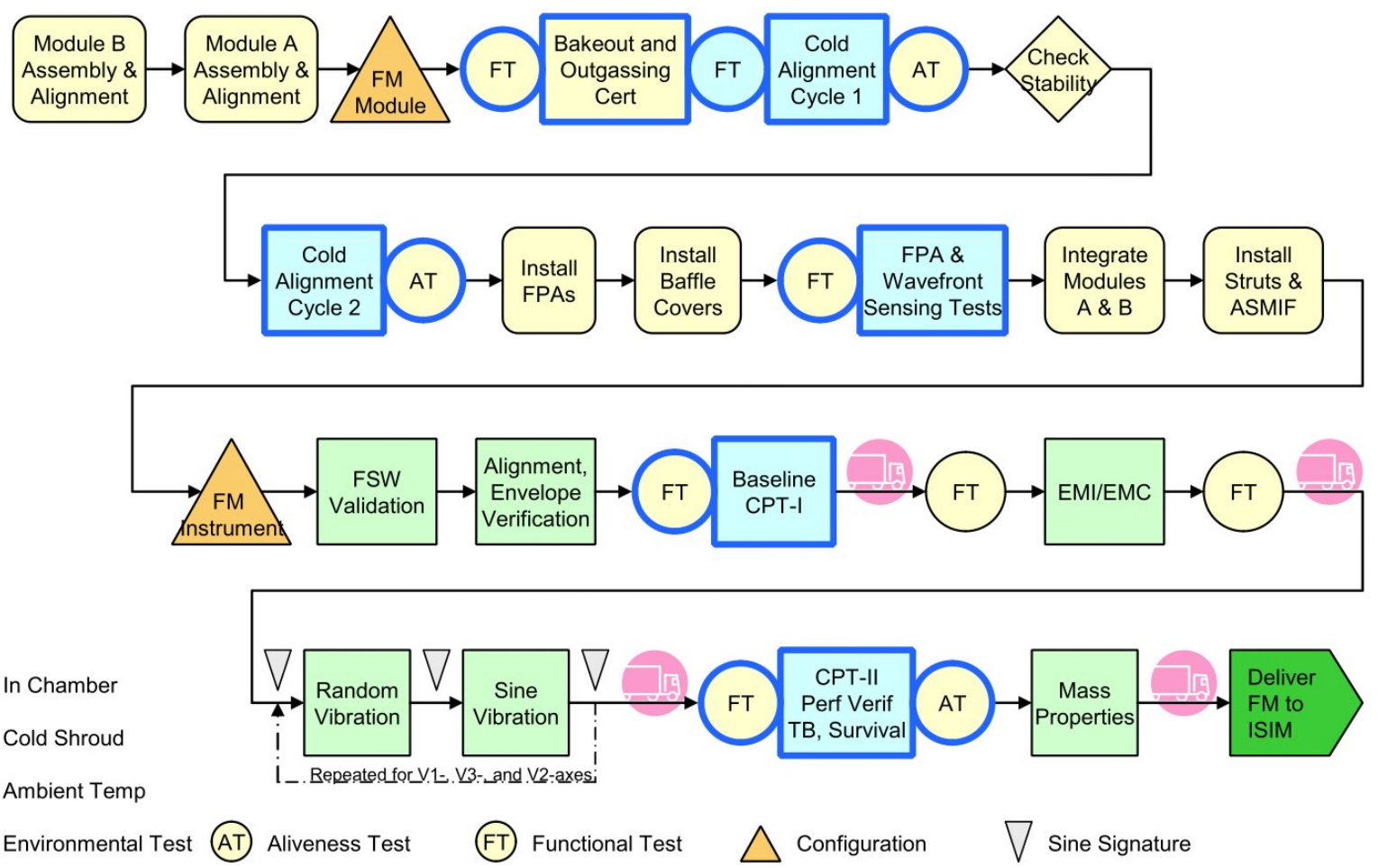

Figure 8. Schematic of the NIRCam FM test sequence.

number of remaining subsystem deliveries and the complexities of the integrated testing, the schedule discussed above may need to be revised before NIRCam is delivered.

\section{REFERENCES}

[1] Gardner, J. P., Mather, J. C., Clampin, M., Doyon, R., Greenhouse, M. A., Hammel, H. B., Hutchings, J. B., Jakobsen, P., Lilly, S. J., Long, K. S., Lunine, J. I., McCaughrean, M. J., Mountain, M., Nella, J., Rieke, G. H., Rieke, M. J., Rix, H., Smith, E. P., Sonneborn, G., Stiavelli, M., Stockman, H. S., Windhorst, R. A., and Wright, G. S., "The James Webb Space Telescope," Space Science Reviews 123, 485-606 (Apr. 2006).

[2] Contos, A. R., Acton, D. S., Atcheson, P. D., Barto, A. A., Lightsey, P. A., and Shields, D. M., "Aligning and maintaining the optics for the James Webb Space Telescope (JWST) on-orbit: the wavefront sensing and control concept of operations," in [Society of Photo-Optical Instrumentation Engineers (SPIE) Conference Series], Society of Photo-Optical Instrumentation Engineers (SPIE) Conference Series 6265 (July 2006).

[3] Shi, F., King, B. M., Sigrist, N., and Basinger, S. A., "NIRCam Long Wavelength Channel grisms as the Dispersed Fringe Sensor for JWST segment mirror coarse phasing," in [Society of Photo-Optical Instrumentation Engineers (SPIE) Conference Series], Society of Photo-Optical Instrumentation Engineers (SPIE) Conference Series $\mathbf{7 0 1 0}$ (Aug. 2008).

[4] Krist, J. E., Beichman, C. A., Trauger, J. T., Rieke, M. J., Somerstein, S., Green, J. J., Horner, S. D., Stansberry, J. A., Shi, F., Meyer, M. R., Stapelfeldt, K. R., and Roellig, T. L., "Hunting planets and observing disks with the JWST NIRCam coronagraph," in [Society of Photo-Optical Instrumentation Engineers (SPIE) Conference Series], Society of Photo-Optical Instrumentation Engineers (SPIE) Conference Series 6693 (Sept. 2007).

[5] Greene, T., Beichman, C., Eisenstein, D., Horner, S., Kelly, D., Mao, Y., Meyer, M., Rieke, M., and Shi, F., "Observing exoplanets with the JWST NIRCam grisms," in [Society of Photo-Optical Instrumentation Engineers (SPIE) Conference Series], Society of Photo-Optical Instrumentation Engineers (SPIE) Conference Series 6693 (Sept. 2007). 
[6] Rieke, M. J., Baum, S. A., Beichman, C. A., Crampton, D., Doyon, R., Eisenstein, D., Greene, T. P., Hodapp, K., Horner, S. D., Johnstone, D., Lesyna, L., Lilly, S., Meyer, M., Martin, P., McCarthy, Jr., D. W., Rieke, G. H., Roellig, T. L., Stauffer, J., Trauger, J. T., and Young, E. T., "NGST NIRCam Scientific Program and Design Concept," in [Society of Photo-Optical Instrumentation Engineers (SPIE) Conference Series], J. C. Mather, ed., Society of Photo-Optical Instrumentation Engineers (SPIE) Conference Series 4850, 478-485 (Mar. 2003).

[7] Burriesci, L. G., "NIRCam instrument overview," in [Society of Photo-Optical Instrumentation Engineers (SPIE) Conference Series], J. B. Heaney \& L. G. Burriesci, ed., Society of Photo-Optical Instrumentation Engineers (SPIE) Conference Series 5904, 21-29 (Aug. 2005).

[8] Somerstein, S. F. and Truong, G. D., "NIRCam optical calibration sources," in [Society of Photo-Optical Instrumentation Engineers (SPIE) Conference Series], J. B. Heaney \& L. G. Burriesci, ed., Society of Photo-Optical Instrumentation Engineers (SPIE) Conference Series 5904, 57-65 (Aug. 2005).

[9] Beichman, C. A., Krist, J., Trauger, J. T., Greene, T., Oppenheimer, B., Sivaramakrishnan, A., Doyon, R., Boccaletti, A., Barman, T. S., and Rieke, M., "Imaging Young Giant Planets From Ground and Space," Publications of the Astronomical Society of the Pacific 122, 162-200 (Feb. 2010).

[10] Krist, J. E., Balasubramanian, K., Muller, R. E., Shaklan, S. B., Kelly, D. M., Beichman, C. A., Serabyn, E., Wilson, D. W., Trauger, J. T., Echternach, P. M., Mao, Y., and Liewer, K. M., "The JWST/NIRCam coronagraph flight occulters," in [Society of Photo-Optical Instrumentation Engineers (SPIE) Conference Series], Society of Photo-Optical Instrumentation Engineers (SPIE) Conference Series 7731 (2010).

[11] Gully-Santiago, M., Jaffe, D. T., Wang, W., Dean, C. P., Kelly, D., Greene, T. P., and Bacon, J. W., "High-performance silicon grisms for $1.2-8.0 \mu \mathrm{m}$ : detailed results from the JWST-NIRCam devices," in [Society of Photo-Optical Instrumentation Engineers (SPIE) Conference Series], Society of Photo-Optical Instrumentation Engineers (SPIE) Conference Series 7739 (2010).

[12] Smith, R., Rahmer, G., Bonati, M., and Zavodny, M., "Image persistence in 1.7 $\mu \mathrm{m}$ cut-off HgCdTe focal plane arrays for SNAP," in [Nuclear Science Symposium Conference Record, 2007, IEEE], Nuclear Science Symposium Conference Record 3, 2236 - 2245 (Nov. 2007). 